\title{
Similarity and contrast in memory for relations
}

\author{
IAN BEGG \\ McMaster University, Hamilton, Ontario L8S 4KI, Canada
}

\begin{abstract}
The paper reports four experiments concerned with memory for sets of categorically related or unrelated nouns, as a function of the extent to which initial processing focused on categorical or distinctive features of the nouns. The general finding is that distinctive features are valuable for later intracategory discrimination in recall and recognition, but categorical features are not, as judged by cued recall, recognition, measures of association between items, and categorical intrusion errors. A theoretical framework, based on the diagnostic value of features for various classifications, is offered as an account of the results.
\end{abstract}

The relation between retention and the processing of words during study has been the subject of many investigations since the appearance of Craik and Lockhart's (1972) levels-of-processing framework. The results of those investigations demonstrate that different types of processing yield different levels of memory performance. However, it is not always clear on theoretically specified grounds whether two particular processing tasks differ in a way that affects retention. The present paper offers a feature-sampling account of encoding, which allows specification of the informational content of memory traces resulting from particular study tasks, and a theoretical account of retrieval, which specifies the manner in which trace composition interacts with retrieval demands to affect performance. The fundamental assumption of the present account, shared with the levels account, is that memory for an event is determined by initial processing of the event. More specifically, the trace of an event is assumed to be the set of information active in the cognitive system at the termination of initial processing. Given this assumption, the goal of the encoding theory is to specify the active set, since that set is the memory trace. The goal of the retrieval theory is to specify the manner in which information present in the retrieval environment reactivates the set, and the manner in which the reactivated set is used to generate responses. The question of whether two study tasks will result in differential retention thus has no absolute answer; if the traces differ in ways that are relevant for retrieval demands, there will be differential memory performance.

By the present account, encoding is a reasonably efficient classificatory process. For example, "beer" is

Reprints can be obtained from Ian Begg, Department of Psychology, McMaster University, Hamilton, Ontario L8S 4K1, Canada. Helpful comments were offered at every stage of the research by members of the F. T. Bacon Society (Grant Harris, John Mitterer, and Douglas Upfold), and by Larry Jacoby. The research was funded by Grant A8122 from the National Research Council of Canada. The manuscript was prepared while the author was a Visiting Associate Professor at the University of Toronto. potentially classifiable in an unlimited number of ways, such as "alcoholic," "liquid," "desirable," "fattening," and so on. However, in any particular context, a smaller subset of classifications is sufficient for unambiguous specification of the item. The basic knowledge concerning an item familiar to the subject has been described by Tversky (1977) as a set of features, with "feature" a general term denoting any piece of knowledge. In any task involving an item, "we extract and compile from our data base a limited list of relevant features on the basis of which we perform the required task" (Tversky, 1977, p. 329). The relevance of any feature depends on the diagnostic value of that feature for the required classification. For example, the feature "alcoholic" has little value within the set of alcoholic beverages because it is shared by all the members, but it has considerable value in distinguishing any one of those members from members of other sets, such as animals or furniture. Let us define encoding as feature sampling, or the compilation of a list of relevant features, with the likelihood that any particular feature is in the resultant sample a function of the diagnostic value of that feature. It then follows that the trace of an event is the sample of features compiled during encoding.

As an illustration, consider a task in which subjects list similarities between "beer" and "wine." Features relevant for the task are those shared by the items, such as "alcoholic," "intoxicant," "beverage," and so on. Thus the trace of the event "beer-wine" will consist of features common to the items comprising the event. Such features are of value for the task, but are of less value for performing other tasks that might be required by retrieval demands. For instance, the features would be useful in deciding whether "beer" or "dog" was in the list, but would be less useful for a decision regarding which of "beer" or "vodka" occurred. Since no feature is of value for discriminating among items that share it, the domain of value for any given feature is specifiable. In sum, the encoding theory defines trace composition as the sample of features resulting from initial processing of an event, and thereby specifies the domain of retrieval 
decisions for which the trace will be of value. The retrieval theory that follows specifies the demands imposed by the retrieval environment and thus provides information regarding whether those demands fall within or exceed the domain in question.

The retrieval task of interest here is cued recall, in which the subject is provided with some information from which to provide verbal responses. By the present account, successful performance requires that the cue be encoded, that the encoded cue contact or access its parent trace, and that the trace contain or provide sufficient data to allow response production (cf. Begg, 1978; Martin, 1967). Recall could fail because a cue contacts the wrong trace or none at all, or because a contacted trace leads to production of the wrong response or none at all. By the present encoding theory, the memory system, after study, contains some number of traces, each of which is a feature sample. An encoded cue is also a feature sample; the likelihood that a cue will contact a trace is a function of the degree of distinctive similarity between the two samples of features (cf. Asch, 1968, 1969). According to Tversky (1977), such distinctive similarity depends on two factors; similarity increases with the number of features common to the two samples and decreases with the number of features common to other samples. Thus, even if a trace and cue share many features, those features will be of little value if they are also shared with other traces or cues. Trace contact by a cue is maximal if the cue features are shared by the appropriate trace and no others. In contrast, a cue will be of little value for trace discrimination if the cue features are common to several traces, and a trace will be of little value for discrimination among potential cues if the trace features are common to several cues.

The likelihood that a cue will contact the appropriate trace, then, depends on the degree of distinctive similarity of the cue-trace relation. That relation can only be assessed with reference to the traces from which the target is to be discriminated and the cues from which the appropriate target is to be discriminated. It is clear that contact will suffer if changes in task demands reduce cue-trace similarity; for example, if initial encoding is accomplished by listing features that characterize a cognitive category, retrieval decisions will be difficult if the subject is required to discriminate among category members, since each candidate will share the trace features. Factors influencing contact will be addressed further in the introductions to the experiments to be reported shortly. For the present, the first stage of retrieval is trace contact, in which an encoded cue contacts a memory trace.

The second stage of retrieval is response production, in which the contacted trace serves as the source of overt responses. There has been considerable debate récently regarding whether response production is mediated by permanent knowledge, as proposed by generation-recognition theories (e.g., Bahrick, 1970; Martin, 1975), or is mediated only by the contents of the trace of the initial event (e.g., Tulving \& Thomson, 1973; Watkins \& Tulving, 1975). By the present account, response production is mediated by permanent knowledge. However, results would conform to the expectations of Tulving and his associates in the special case in which trace features are sufficiently precise to specify one verbal item. More generally, trace features are common to a family of items, making it necessary to discriminate among family members on the basis of which bears the most distinctive similarity to the trace. Simply put, a contacted trace is both a cue for generating response candidates and the criterion against which the adequacy of those candidates is assessed. Consequently, response confusions should occur if related candidates are made salient by the retrieval task.

These hypotheses will be summarized before presenting the experiments in detail. An event is encoded by compiling a sample of features sufficient to classify the event in whatever way is required by the study task. The resultant sample is the memory trace of the event, and its featural composition constrains the circumstances in which the trace can be contacted and used later on. A retrieval cue, itself encoded as a feature sample, must be recognized; that is, the cue must contact the appropriate trace in order for successful recall. The likelihood of contact depends on the distinctive similarity of the cue-trace relation. A contacted trace is available for use, both as a cue for accessing response candidates from permanent knowledge and as a criterion for discriminating among those candidates. The adequacy of this account is assessed in a series of experiments. The general procedure for each experiment involves presenting prominent exemplars of common categories (Battig \& Montague, 1969), biasing processing of those items toward general categorical features or more unique features, and manipulating retrieval demands to require global categorical discriminations or more precise discriminations among relatives. In each case, the account predicts specific interactions between conditions of study and test.

\section{EXPERIMENT 1}

The first experiment replicates and extends an important experiment by Epstein, Phillips, and Johnson (1975). Epstein et al. presented lists comprising either pairs of categorically related items, such as "beerwine," or categorically unrelated items, such as "beerdog." Subjects listed either similarities or differences between the members of each pair. In later cued recall, similarity processing exceeded contrastive processing for unrelated pairs, but for related pairs, contrastive processing proved superior. Note in passing that in each case the more difficult processing task yielded superior 
retention; however, since Craik and Tulving (1975) ruled out difficulty as a causal factor in determining retention after different processing tasks, a more satisfactory explanation is needed. Consider the explanation that follows from the present account.

To begin, a related pair, "beer-wine," consists of two sategory coordinates. Similarity processing biases sampling to include features common to the two items; such features are also likely to be common to other members of the parent category. The trace is therefore likely to contain features such as "alcoholic beverage." However, contrastive processing biases sampling toward features of one item but not the other; such features, by definition, cannot be categorical. The trace will contain features like "amber liquid" or "cork vs. bottle cap." In the experiment under consideration, each pair was categorically unrelated to any other pair, and each cue was unrelated to each other cue. Consequently, trace-contact differences should be minimal, since either categorical features or features unique to the studied event are of sufficient diagnostic value to allow the rather global categorical decision required during retrieval. However, there should be substantial differences in response production. The "beer-wine" trace following contrastive processing contains features not shared by other alcoholic beverages, and therefore allows a relatively easy decision regarding which to produce. On the other hand, even if the cue "beer" contacts the "beer-wine" trace resulting from similarity processing, there remains some decision regarding whether "wine" is more related to the trace than potential candidates such as "vodka."

It follows that contrastive processing should yield better cued recall of related pairs than should similarity processing. The retention difference should reflect response production rather than trace contact, which should be virtually perfect because of the triviality of cue and trace discrimination in the task. In order to measure response production independently of cued recall, a free recall task was included in the present experiment. If the account is correct, the likelihood that "wine" will be recalled in an output position adjacent to "beer," given recall of "beer," should favor contrastive processing as much as does the likelihood that "wine" will be recalled, given "beer" as a cue; further, in the limiting case in which contact is perfectly likely in cued recall, the conditional free recall and cued recall probabilities should be equal.

With unrelated pairs, such as "beer-dog," we can also expect similarity processing to focus on features common to the items, and contrastive processing to focus on features not common to both. However, the relation of such features to the parent categories is quite different. Features shared by "beer" and "dog," such as "likable," "has a head," "cheap," and so on, cannot be defining features of either parent category, while features that distinguish "beer" from "dog," such as "alcohol vs. animal," might be shared with members of either category, such as "wine" or "cat." Consequently, although either set of features is adequate for trace contact in the case where traces and cues are categorically unrelated, the features common to the unrelated items will be of more value for discriminating among potential response candidates than will the features by which the target items differ. Clearly, then, we should expect just the opposite differences between processing tasks with unrelated as with related items.

\section{Method}

Subjects. Forty student volunteers were paid $\$ 2 / \mathrm{h}$, with 10 students in each of four conditions. Subjects were tested individually in sessions lasting $.75 \mathrm{~h}$.

Materials. The study list consisted of 10 pairs of related nouns, 10 pairs of unrelated nouns, and an additional 40 pairs of unrelated filler nouns. The fillers were selected from Paivio, Yuille, and Madigan (1968) to cover a wide range of imagery and frequency values, and they were randomly paired. The 20 critical pairs consisted of prominent exemplars of culturally shared categories. Two of the five most frequent exemplars were chosen from each of 30 categories (Battig \& Montague, 1969). Both members were retained from 10 of the categories, yielding 10 related pairs. One member from each of the remaining 20 categories was retained, with random pairing yielding 10 unrelated pairs. Each of the 60 pairs was printed on a separate index card.

Procedure. Each subject first studied the 20 critical pairs, then the 40 fillers; the purpose of the fillers was to extend the retention interval for the critical pairs. Half the subjects used each of two processing tasks. In a similarities task, subjects listed ways in which the members of each pair were similar to each other; in a contrastive task, subjects listed ways the members differed. For the critical pairs, presentation was self-paced, with subjects listing either three similarities or three contrasts. For the fillers, presentation was paced at $15 \mathrm{sec} / \mathrm{pair}$, to equalize the retention interval for the critical pairs.

Retention of the critical pairs was assessed immediately following study. For half the subjects, the test was a cued recall test, $3 \mathrm{~min}$ in duration, with one member of each pair as a cue. For the other subjects, retention of the critical pairs was assessed by a 3-min free recall test; following the test, these subjects free recalled fillers for $6 \mathrm{~min}$, then were administered a cued recall test for the critical items.

Overall, subjects studied pairs of related or unrelated category exemplars by listing similarities or differences between pair members. Retention was assessed by immediate cued recall, or by immediate free recall and delayed cued recall.

\section{Results and Discussion}

Throughout the experiments, $\alpha$ was set at .05 . Means are presented as proportions with standard deviations in parentheses, although analyses were conducted on the numbers correct. In Experiment 1, immediate cued recall exceeded delayed cued recall, but averaged data are presented, since delay interacted with no other variables.

Related pairs. The most critical contrast of the experiment is between processing tasks for the related pairs. In each analysis, contrastive processing exceeded similarity processing, with very high levels of perform. ance throughout, despite the interpolated study of 
filler items. In cued recall, contrastive processing led to recall of $.97(.06)$ of the responses, compared to .91 (.11) for similarity processing $[\mathrm{t}(39)=2.43]$. In free recall, the proportion of recalled items correctly paired also favored contrastive processing, with means of $.98(.06)$ and $.87(.15)[\mathrm{t}(19)=2.13]$; for the same subjects, delayed cued recall means were $.97(.05)$ and $.84(.11)[\mathrm{t}(19)=3.20]$, which are quite similar to the conditional measures, as expected.

The results for related pairs are easy to summarize. Contrastive processing exceeds similarity processing in cued recall, and in the probability, given recall of one pair member, that the other will appear in a contiguous output position. Since the conditional recall probabilities are approximately equal to cued recall probabilities, we can conclude that cue-to-trace contact was not an important factor in recall. That is, the cue-contact stage of recall required no intracategory discrimination, with the result that even factors diagnostic only for betweencategories discrimination are adequate for the task. However, since response production is also dependent on trace-response distinctive similarity, features relevant for performance are those with diagnostic value in distinguishing among category members; features distinctive to the studied members are of more value for this purpose than common features. Consequently, contrastive processing exceeds similarity processing for related pairs in cued recall, with the bulk of the effect attributable to differential trace utility in response production.

Unrelated pairs. Results for unrelated pairs were opposite to those for related pairs. Cued recall favored similarity processing, with means of $.82(.17)$ and .32 (.18) $[\mathrm{t}(19)=9.01]$. In free recall, because of several zero cells, conditional recall probabilities are presented as group statistics rather than means over individual subjects. For similarity processing, .73 of recalled words were correctly paired, with mean delayed cued recall of .73 (.19); for contrastive processing, .44 of recalled words were correctly paired, with the same subjects recalling .26 (.19) of the responses in cued recall.

In summary, unrelated pairs produce higher cued recall and conditional free recall following similarity processing than following contrastive processing. Thus both common features and distinctive features of the unrelated members are of diagnostic value in betweencategories discrimination, but the common features are of more value in the within-categories discrimination required in response production, because they are not shared with other family members.

\section{EXPERIMENT 2}

The purpose of Experiment 2 was to control more precisely the initial processing task, in order to assess predictions from the present account. A pair such as "beer-wine" can be processed in terms of features unique to the pair, as well as in terms of features characteristic of the parent category or unique to either term. In the second experiment, subjects received related triplets, such as "beer-wine-scotch," with the task of comparing the first two terms to the third one. In the similarity condition, subjects were instructed to list ways that "beer" and "wine" were similar to each other and to "scotch"; such instructions should bias sampling of categorical features that should be valuable for categorical discrimination but not for discrimination among category relatives. In the contrastive condition, subjects were instructed to list ways "beer" and "wine" were similar to each other but not to "scotch"; although such processing does not require the first two terms to be contrasted with each other as in Experiment 1, features adequate for the requirement should also be adequate for distinguishing the presented items from other members of the parent category.

Following processing, retention was assessed by cued recall, with either the first term (beer) or the third term (scotch) the cue for the remaining two terms. Since each triplet in the task was from a separate category, cue-trace contact should again be relatively trivial. Consequently, the results should be quite similar to those for related pairs in Experiment 1. The overall level of cued recall should favor contrastive over similarity processing, regardless of which term is the cue. Further, because two terms were always to be recalled, it is possible to assess the degree of relation between the two recalled terms. For example, with "beer" as the cue, the probability that "wine" will be recalled, given that "scotch" is recalled, should then equal the probabiliy that "wine" will be recalled given "scotch" as a cue; in short, conditional recall of some item, $\mathrm{Y}$, given recall of some other item, $X$, should be equal to unconditional cued recall of $\mathrm{Y}$ by $\mathrm{X}$, since contact should be quite automatic.

Experiment 2 also examines intrusion errors, which should reflect confusion in whatever discriminations are required for performance. There should be relatively few intralist intrusions in either condition, since all other list items were categorically distinct from the particular cue or trace in question. However, there should be more extralist intrusions of categorical relatives following similarity processing than following contrastive processing if such relatives are generated and edited against the trace; the trace following similarity processing contains features also shared by unpresented relatives, making the recognition decision less precise than in the contrastive case.

In a second major condition, the third terms of all related triplets were randomly interchanged, yielding triplets with a related pair, such as "beer-wine," and an odd term, such as "dog." Again, subjects either contrasted the first two terms with the third term or listed ways all three terms were similar, and again, either the first or third term appeared as a recall cue 
for the remaining two terms. In general, we should expect similarity processing to yield superior retention because the sampled features are less likely than those following contrastive processing to be characteristic of the parent categories. However, the expectations can be made more precise. In all comparisons considered to this point, trace contact has been relatively easy, because one, and only one, cue is categorically related to any given trace. In the present case, however, each triplet is related to two others in the list; "beer-winedog" is related to whichever triplet contains "scotch" as an odd term and to whichever triplet contains two animals as the related members. Consequently, there should now be evidence of cues contacting the wrong traces. Intralist intrusions should be more common than in the related triplets, and more so if the third rather than the first term is the cue, while extralist intrusions should be less discrepant.

As with related triplets, the important predictions concern the level of cued recall and the measures of association between terms. Because contact likelihood is reduced with the triplets containing an odd term, we should no longer expect conditional recall probabilities to equal cued recall probabilities, but rather to exceed them to the extent that contact is less likely. However, differences in cued recall between instructions should be directionally mirrored in conditional probabilities. Two patterns are expected. First, if recall is across a category boundary, similarity processing should exceed contrastive processing. Thus, with "dog" as a cue for "beer" and "wine," with "beer" as a cue for "dog," or with the degree of association between "wine" and "dog" with "beer" as the cue, similarity processing should prove the better procedure. On the other hand, with measures that are not across category boundaries, there is no reason to expect any instructional effect; thus, with "beer" as a cue for "wine," or the association between "beer" and "wine" with "dog" as the cue, no retention difference should obtain.

\section{Method}

Subjects. Forty student volunteers were paid $\$ 2 / \mathrm{h}$, and there were 10 in each of four conditions. Subjects were tested individually in sessions lasting $1 \mathrm{~h}$.

Materials. Study material in all conditions consisted of 72 nouns, 3 from each of 24 conceptual categories (Battig \& Montague, 1969). For the ABC list, the list was presented as 24 triplets, each containing three related items. For the ABX list, the $\mathrm{C}$ terms were randomly interchanged, again yielding 24 triplets. Each triplet was printed on a separate index card.

Procedure. Half the subjects studied each list, with half of each group processing in a similarities task, half in a contrastive task. In similarities, subjects reported one way in which $A$ and $B$ were similar to each other and to $C$ or $X$. In contrasts, subjects also listed a way in which $A$ and $B$ were similar to each other, but that distinguished them from $C$ or $X$. Following study, retention was assessed by intralist cued recall; for half the triplets, A was the cue, while for half, the appropriate third term, $\mathrm{C}$ or $\mathrm{X}$, was the cue. In each case, subjects were to recall the two missing words, with 5 min allowed for the task.

\begin{tabular}{|c|c|c|c|c|c|}
\hline & & \multicolumn{4}{|c|}{ Processing } \\
\hline & & \multicolumn{2}{|c|}{ Similarity } & \multicolumn{2}{|c|}{ Contrastiv } \\
\hline & & Mean & SD & Mean & SI \\
\hline & & \multicolumn{4}{|c|}{ Related Triplets ( $A B C)$} \\
\hline Cued by $\mathrm{C}$ & $\begin{array}{l}\text { Recall of A } \\
\text { Recall of B } \\
\text { Association }(A, B)\end{array}$ & $\begin{array}{l}.80 \\
.74 \\
.80\end{array}$ & $\begin{array}{l}.12 \\
.16 \\
.14\end{array}$ & $\begin{array}{l}.94 \\
.95 \\
.98\end{array}$ & $\begin{array}{l}.05 \\
.04 \\
.04\end{array}$ \\
\hline Cued by $\mathrm{A}$ & $\begin{array}{l}\text { Recall of } B \\
\text { Recall of } C \\
\text { Association }(B, C)\end{array}$ & $\begin{array}{l}.82 \\
.79 \\
.83\end{array}$ & $\begin{array}{l}.09 \\
.10 \\
.10\end{array}$ & $\begin{array}{l}.92 \\
.93 \\
.96\end{array}$ & $\begin{array}{l}.08 \\
.06 \\
.06\end{array}$ \\
\hline & & \multicolumn{4}{|c|}{ Odd Triplets (ABX) } \\
\hline Cued by $\mathrm{X}$ & $\begin{array}{l}\text { Recall of } A \\
\text { Recall of } B \\
\text { Association }(A, B)\end{array}$ & $\begin{array}{l}.55 \\
.58 \\
.86\end{array}$ & $\begin{array}{l}.18 \\
.18 \\
.13\end{array}$ & $\begin{array}{l}.19 \\
.18 \\
.86\end{array}$ & .09 \\
\hline Cued by $A$ & $\begin{array}{l}\text { Recall of } B \\
\text { Recall of } X \\
\text { Association }(B, X)\end{array}$ & $\begin{array}{l}.60 \\
.40 \\
.59\end{array}$ & $\begin{array}{l}.11 \\
.26 \\
.27\end{array}$ & $\begin{array}{l}.64 \\
.05 \\
.08\end{array}$ & .19 \\
\hline
\end{tabular}

\section{Results and Discussion}

Cued recall. The basic results are presented in Table 1, with the dependent measure the likelihood a given term will be recalled when another term is the cue. First, consider the related triplets in the top half of the table. For each of the four recall measures, contrastive processing, with an overall mean of .94 , exceeded similarity processing, with an overall mean of .79; the four $t(19)$ values each exceeded 2.55. Thus, the advantage for contrastive over similarity processing of related items does not require that the cue and to-beremembered items have been contrasted with each other, as in Experiment 1, but extends to the case where the cue and to-be-remembered item have been contrasted with some other related term. Note, as well, that the degree of association between $\mathbf{A}$ and $\mathrm{B}$, measured as the likelihood that one is recalled given recall of the other, approximates the likelihood that one is recalled given the other as a cue, with an equivalent finding for $B$ and $C$; in each case, contrastive processing exceeded similarity processing, with both $t(19)$ values exceeding 2.20. As in Experiment 1, trace contact seems not to have been a problem with a relatively short list of categorically distinctive sets of items.

Next, consider the odd triplets in the bottom half of the table. With the odd term $(X)$ as the cue, recall favored similarity processing over contrastive processing; both $t(19)$ values exceeded 5.68. With the first term (A) as the cue, recall of the odd term favored similarity processing $[\mathrm{t}(19)=4.07]$, but recall of the member that was categorically related to the cue showed no difference $[t(19)=.55]$. Note that the degree of association between the two related members, $A$ and $B$, was equal after both processing tasks, and at quite a high level relative to cued recall of one term by the other; 
thus, the processing tasks do not differ in either the likelihood of contact or the response production given contact, although contact was not perfectly likely, since the conditional measures were higher than the marginal measures of recall. Also note that the odd term was more highly associated with the B term following similarity processing than following contrastive processing $[t(19)=5.40]$. Finally, note that the odd term was less likely to be recalled with $A$ as the cue than was the A term to be recalled given the odd term as the cue, for each processing task.

Errors. The number of extralist cate'zorical intrusion errors, of 48 possible, was analyzed by a 2 by 2 by 2 analysis of variance, with task and triplet type as independent factors and cue (first or third term) as a repeated factor. More errors occurred following similarity processing than following contrastive processing $[\mathrm{F}(1,36)=17.7]$ and with $\mathrm{ABC}$ rather than $\mathrm{ABX}$ triplets $[F(1,36)=9.67]$; the interastion between the two variables was marginally reliable $[\mathrm{F}(1,36)=4.05$, $p=.05]$. Similarity processing of $A B C$ triplets had the highest intrusion rate, with a mean of 2.4 (1.5), compared to similarity processing of $\mathrm{ABX}$ triplets at 1.0 (1.4), contrastive processing of $\mathrm{ABC}$ triplets at .7 (.8), and contrastive processing of $\mathrm{ABX}$ triplets of .4 (.5). Thus there is evidence of intracategory confusion, especially for similarity processing of $\mathrm{ABC}$ triplets.

Second, the number of intralist intrusions, or mispairings, was analyzed by a 2 by 2 by 2 analysis of variance with factors as above. Intrusions were more frequent with $\mathrm{ABX}$ than with $\mathrm{ABC}$ triplets $[\mathrm{F}(1,36)=24.4]$ and with the third term as a cue than with the first $[F(1,36)=23.3]$, and there was a large interaction between the variables $[F(1,36)=22.1]$. Errors were extremely rare with $\mathrm{ABC}$ triplets, with no condition exceeding 3 (.7). However, errors were more common with $\mathrm{ABX}$ triplets, and more so if $\mathrm{X}$ rather than $\mathrm{A}$ was the cue, with means of $5.6(4.5)$ and $1.9(2.4)$

\section{Summary and Conclusions}

The most important results of Experiments 1 and 2 pertain to the advantage of contrastive over similarity processing of related items in later cued recall, and to the fact that measures of association between a cue and its response are about the same, assessed in cued recall of one by the other, or in terms of output adjacency of one to the other. By the present account, the differences stem from the nature of the memory traces following the different processing tasks. Features common to the list items are also likely to be common to categorical relatives, while features that distinguish list items from each other are less likely to be categorical. Either type of feature is adequate for distinguishing members of the category in question from members of other general categories, with the result that cue-to-trace contact should be virtually error free, as indexed by (1) the equality of conditional recall to cued recall, and (2) the paucity of intralist intrusion errors. Contact likelihood will be addressed more directly in Experiment 3, in which categorically related distractors are added to the list of cues. With categorically distinctive traces of the type inferred in the first two experiments, the main reason for recall differences should be the greater difficulty of recognition decisions among related response candidates following similarity processing. Such difficulty is manifested by decreased cued recall, decreased association, and increased incidence of extralist intrusions, relative to the contrastive conditions.

The findings are equally strong for retention differences favoring similarity over contrastive processing if the items are categorically unrelated to begin with. However, the differences are of less interest theoretically because most theoretical accounts would expect a similarity advantage, since such processing requires coprocessing the items rather than processing them separately (cf. Begg, 1978). The present account does gain some advantage, however, in successfully predicting relations between cued recall and conditional free recall, and intralist intrusions in addition to simple recall differences. Nonetheless, the remainder of the paper will concern the more interesting related items.

\section{EXPERIMENT 3}

The purpose of Experiment 3 was to investigate further the retention of related pairs initially processed by similarities or contrasts. In the previous experiments, trace contact was assumed to be quite easy, with the result that all retention differences should reflect response production, with the more difficult recognition decision among response candidates occurring after similarity processing. In order to vary the difficulty of trace contact in addition to response production, Experiment 3 manipulated the nature of the retrieval cues, with three conditions. The first condition was a standard intralist cued recall test, as in Experiment 1; contrastive processing should exceed similarity processing, with the difference attributable to differential response production. The second condition was a modified intralist cued recall task, in which the cue list contained categorically related distractors in addition to list cues. Since cue recognition now requires discrimination among categorical relatives, the similarity condition should suffer relative to the contrastive condition in recall. The additional decrement in recall because of the distractors should reflect differences in trace contact, while the difference in the first condition should reflect differences in response production. Thus, if the results are scored to reflect (1) cue recognition, or contact, and (2) response recall given cue recognition, or response production, both measures should favor contrastive processing.

In the third condition, category labels were provided as extralist cues for recall of both studied members of 
each category. Since all list items are highly prominent members of their parent categories, provision of the label should guarantee the availability of the list items as responses, but should also increase the availability of other candidates. Again, no new problems should occur for the contrastive condition, because the traces are specifically related to the studied items. However, the increase in availability of incorrect responses should be harmful for the similarity condition. If recall in that condition is conditional on recall of the item serving as the cue in Condition 1, results should again be about equal to the recall means from that condition.

Finally, errors provide a way of assessing another aspect of the present account. If the trace is the criterion against which cues are recognized and the adequacy of candidates is assessed, then the likelihood that a related item is intruded in recall should equal the likelihood that a related item is falsely recognized. This prediction will be assessed.

\section{Method}

Subjects. Seventy-eight student volunteers were paid $\$ 2 / \mathrm{h}$; 13 subjects, tested individually, served in each of six conditions.

Materials and Procedure. The study material for all subjects was 24 pairs of related nouns, each pair from a different category (Battig \& Montague, 1969), and each printed on a separate index card. Half the subjects listed two ways the pair members contrasted with each other, and half listed two ways the members were similar. Following study, each subject engaged in $40 \mathrm{~min}$ of intervening activity, during which time 80 filler pairs were studied and recalled; the intervening period was introduced in an attempt to lower performance from the very high levels obtained in Experiments 1 and 2. After the interpolated activity, retention was assessed by one of three cued recall tests. Two of the tests involved intralist cues, with one member of each pair as a cue for its associate; in one condition, the 24 cues were simply presented as in Experiment 1, while in the other, the 24 cues were randomly interspersed with 24 distractor nouns, 1 from each of the 24 categories, with subjects required both to circle cues that were old and to recall the associates. The third condition involved extralist cues; the 24 category labels appeared as cues, and subjects attempted to recall both pair members for each cue.

Overall, subjects processed related pairs in terms of shared or contrastive characteristics, then were tested by cued recall, by one of three tests. One test was simply intralist cued recall, a second was also intralist cued recall but required cue discrimination prior to recall, and a third was extralist cued recall with category labels as cues.

\section{Results and Discussion}

In each of the three conditions, cued recall levels were higher following contrastive processing than following similarity processing. For the control condition, the respective means were $.91(.09)$ and .81 (.17); with intralist cues and distractors, the means were $.85(.14)$ and $.58(.22)$; with extralist cues, the means were $.85(.12)$ and $.66(.16)$. Performance did not differ reliably over tasks with contrastive processing $[F(2,36)=1.14]$, although the tasks differed with similarity processing $[\mathrm{F}(2,36)=4.88]$; based on a post hoc $t$ test, the first condition exceeded the other two conditions, which did not differ. Thus, performance in cued recall was better if related pairs, such as "beerwine," were initially processed in terms of contrastive rather than shared semantic knowledge. Further, memory for pairs contrastively processed was relatively impervious to interference produced by adding competing categorically related cues, or by providing general categorical cues rather than the specifically processed cues. Before discussing the results in more detail, some finer grained analyses will be reported.

Components of recall. By the account presented in the introduction, cued recall requires first that the cue be encoded, second that the encoded cue contact the trace of the cue's prior occurrence, and third that the contacted trace provide enough information to allow production of the desired response. In the condition in which categorically related distractors appeared among the cues, it is possible to measure (1) cue recognition, which reflects trace contact, and (2) recall given cue recognition, which reflects the informational value of contacted traces. Contrastive processing exceeded similarity processing on both measures, with respective recognition hit rates of .93 $(.09)$ and $.80(.14)[\mathrm{t}(25)=2.80]$ and conditional recall probabilities of $.91(.13)$ and $.68(.22)[\mathrm{t}(25)=3.15]$. Thus, cues are more likely to contact their parent traces after contrastive processing than after similarity processing, and those traces are more likely to contain information specifically enough related to the response to allow correct decoding, both of which would occur if highly distinctive mumory traces were formed initially. Additionally, conditional recall probabilities can be measured in the condition with extralist cues; because the study material consisted of highly prominent exemplars of parent categories, the provision of the category labels should guarantee the availability of the items as response candidates. Given that the item that served as a cue in the other conditions was recalled, the probability that its associate was recalled as well was .92 (.06) for contrastive processing, which reliably exceeded the value of $.77(.18)$ obtained for similarity processing $[t(25)=2.92]$; the values indeed approximate the level of cued recall in the first conditions, with means of .91 and .81 .

Errors. In the first condition, overt intrusion errors occurred at a level of $.03(.05)$ for contrastive processing and $.11(.06)$ for similarity processing $[\mathrm{t}(25)=3.58]$. With distractors present among the cues, the false recognition rate for categorically related distractors was .04 (.07) for contrastive processing and $.13(.11)$ for similarity processing $[t(25)=2.57]$. That is, the overall intrusion rate was virtually identical to the false recognition rate, which would occur if response candidates from the parent category were generated and edited for output. As further support, the probability of an intrusion given cue recognition yielded comparable values of $.05(.107)$ and $.13(.11)[t(25)=2.07]$ for 
contrastive and similarity processing, respectively. Finally, with extralist cues, intrusion rates were also comparable, with respective means of $.05(.04)$ and .13 (.11) $[\mathrm{t}(25)=2.61]$ for contrastive and similarity processing. That is, the probability of an intrusion in the control task was equal to the probability of an intrusion given cue recognition or given the category label as a cue, and all three values were equivalent to the false recognition rate. In every case, errors were very rare for contrastive processing, but more frequent for similarity processing.

\section{EXPERIMENT 4}

The fourth experiment is primarily of parametric interest. Experiment 3 assessed the influence of distractors among the cues in affecting cue recognition. Experiment 4 assesses response recognition, again with distractors, with the simple expectation that contrastive processing will exceed similarity processing in contact so assessed. Additionally, items were given either one or two study trials.

\section{Method \\ Subjects. Twelve paid volunteers, tested individually, served as subjects. \\ Materials. The study material consisted of 40 pairs of cate- gorically related items, chosen from 40 different categories (Battig \& Montague, 1969). Twenty pairs were each printed on one index card, while 20 were printed on each of two cards; the 60 cards comprised the study list. \\ Procedure. For each card in the deck, the subject listed either one similarity or one difference between the members of ihe pair. For the 20 singly presented pairs, half were processed contrastively, and half in terms of similarities. For the 20 twice- presented pairs, 5 were processed by similarities on both tests. 5 by contrasts, and 10 by one of each, with 5 in each order. Following study, one member of each pair occurred in a recog- nition list, with an equal number of distractors, one from each of the 40 catcgories. After the $3-\mathrm{min}$ recognition test, a cued-recall test was administered, with one member of each pair serving as a cue for recall of the other. There are two points to note. First intervening between study and test was a task about $1 \mathrm{~h}$ in dura- tion, completely unrelated to the present task, again to reduce performance. Second, the recognition targets were the response members of the pairs, although the subjects were unaware of this fact.}

\section{Results and Discussion}

Recognition. Recognition results are presented as hit rates, since an analysis of $\mathrm{d}^{\prime}$ values produced identical results. For once-presented pairs, hit rates for contrastive and similarity processing were $.80(.14)$ and $.68(.17)$; for pairs studied twice by contrasts and twice by similarities, the means were $.95(.09)$ and $.83(.22)$. Thus, twice-presented items were recognized better than once-presented items $[F(1,11)=6.53]$ and contrastive processing exceeded similarity processing $[F(1,11)=11.6]$. For pairs studied once by each processing task, the hit rate was $.94(.10)$.

Recall. Recall performance replicated the results of the first three experiments. For once-presented pairs. recall rates were $.68(.13)$ and $.58(.16)$ after contrastive and similarity processing, respectively, with means of $.88(.13)$ and $.75(.26)$ for pairs processed twice by either strategy. Again, contrastive processing exceeded similarity processing $[F(1,11)=12.3]$, and twice-studied pairs exceeded once-studied pairs $[\mathrm{F}(1,11)=10.1]$. For pairs processed once by each task, the recall level was $.89(.12)$

\section{Summary}

The experiment simply demonstrates recall and recognition advantages for contrastive processing of related pairs over similarity processing of the same pairs.

\section{GENERAL DISCUSSION}

Although the present experiments offer a large quantity of data, the major results are easy to summarize. Retention of related pairs of items depends on the initial processing of those items and on the demands imposed by the retrieval environment. If initial analysis is biased toward features common to the items as opposed to features that differentiate them, retention shows evidence of confusion of the remembered items with related semantic terms. Such terms are relatively likely to be intruded in recall and relatively likely to be falsely recognized as list members. A further consequence of confusion is that both recall and recognition are adversely affected; however, the adverse effect on recognition only holds if the retrieval task requires discrimination on a finer basis than that required in initial processing. Quite simply, features relevant for a global distinction suffer if they are required to serve a more precise requirement. With unrelated terms, the general picture is the reverse; processing for differences is inferior to processing for similarities in later associative performance. The results thus join a growing body of evidence (e.g., Morris, Bransford, \& Franks, 1977) that the effects of differential processing on retention are relative to the manner in which retention is assessed, rather than being generally characteristic of all measures of retention.

The relativistic nature of the results is in keeping with the present relational account, which consists primarily of procedures for specifying trace composition and delimiting the circumstances in which such traces are useful for memory performance. By this account, encoding consists of compiling a sample of features adequate to perform whatever classifications are required initially. That sample is the trace of whatever event initiated processing. It is immaterial whether the event is a word, a pair, or a triplet, since the trace is a psychological unit, not a nominal unit defined physically or linguistically. Accordingly, once a list has been processed, the memory system contains as many traces as there were classification episodes during study. By the present account, all memory performance is mediated by the trace. which must first be contacted and 
then used to produce recall. A cue, itself encoded as a set of features, will contact the trace of its prior occurrence as a function of the degree of distinctive similarity of the cue-trace relation relative to other traces and cues; features appropriate for a given study task are of little value for discriminating among items sharing those features, but of considerable value for discriminating items possessing those features from items not possessing them. Once a trace is contacted, it is available for use as a cue for generating items similar to the trace as response candidates, and for use as a criterion for editing those candidates for adequacy.

The present account bears some similarity to other accounts, but, in total, it is different from each of the others. The determination of trace composition by initial processing is central to Craik and Lockhart's (1972) levels-of-processing framework. However, that account postulates a general dimension correlated with memorability. The present account assumes no such dimension, the closest counterpart being a distinctive similarity relation among cues, traces, and appropriate responses. The present account also assumes, along with generation-recognition theories, that recall can be mediated by permanent knowledge (cf. Martin, 1975), especially if prominent members of common categories are the materials. If Watkins and Tulving (1975) are correct, the trace of the processing episode exists and is used independently of whatever semantic knowledge was used in establishing the trace. Such an account is not contradicted by the present findings concerning contrastive processing of related items, but the high semantic intrusion rate for the same items processed for similarities can only be explained with recourse to semantic mediation. In any event, there is no way to distinguish between semantically mediated recall in the case in which the trace only allows one item and direct production from the trace itself. The semantic view gains an advantage, however, in being able to incorporate the episodic view as a special case.

The general point, regardless of whether the hypothetical trace is conceived as a set of features, attributes, procedures, or whatever, is that retention is a multiply determined event (cf. Begg, 1978), depending on processing, the results of that processing, and later requirements. Despite the relational nature of the various processes, however, quite precise directional predictions can be made by paying close attention to the various demands. It might turn out that quantitative predictions, derived jointly from the retrieval assumptions here and the scaled values of similarity from a theory such as that of Tversky (1977) are reasonably good. However, such a possibility will depend on further research.

\section{REFERENCES}

Asch, S. E. The doctrinal tyranny of associationism: Or what is wrong with rote learning. In T. R. Dixon \& D. L. Horton (Eds.), Verbal behavior and general behavior theory. Englewood Cliffs, N.J: Prentice Hall, 1968.

Asch, S. E. A reformulation of the problem of associations. American Psychologist, 1969, 24, 92-102.

BaHRICK, H. P. A two-phase model for prompted recall. Psychological Review, 1970, 77, 215-222.

Battig, W. F., \& Montague, W. E. Category norms for verbal items in 56 categories: A replication and extension of the Connecticut category norms. Joumal of Experimental Psychology, 1969, 80(3, Part 2).

BEGG, I. Imagery and organization in memory: Instructional effects. Memory \& Cognition, 1978, 6, 174-183.

CRAIK, F. I. M., \& LockHART, R. S. Levels of processing: A framework for memory research. Journal of Verbal Learning and Verbal Behavior, 1972, 11, 671-684.

Craik, F. I. M., \& Tulving, E. Depth of processing and the retention of words in episodic memory. Journal of Experimental Psychology: General, 1975, 104, 268-294.

Epstein, M. L., Phillips, W. D., \& Johnson, S. J. Recall of related and unrelated word pairs as a function of processing level. Journal of Experimental Psychology: Human Learning and Memory, 1975, 1, 149-152.

MARTIN, E. Relation between stimulus recognition and paired. associative learning. Journal of Experimental Psychology. $1967,74,500-505$.

MARTIN, E. Generation-recognition theory and the encoding specificity principle. Psychological Review, 1975, 82, 150-153.

Morris, C. D., Bransford, J. D., \& Franks, J. J. Levels of processing versus transfer appropriate processing. Joumal of Verbal Learning and Verbal Behavior, 1977, 16, 519-534.

Paivio, A., Yuille, J. C., \& Madigan, S. A. Concreteness, imagery and meaningfulness values for 925 nouns. Journal of Experimental Psychology Monograph Supplement, 1968, 76(1, Part 2)

Tulving, E., \& Thomson, D. M. Encoding specificity and retrieval processes in episodic memory. Psychological Review, 1973, 80, 352-373.

Tversky, A. Features of similarity. Psychological Review, 1977. 84. 327-352.

Watkins, M. J., \& Tulving, E. Episodic memory: When recog. nition fails. Journal of Experimental Psychology: General. $1975,104,5-29$.

(Received for publication March 27, 1978; revision accepted August 16, 1978.) 